'Departamento de Economía de la Salud, División de Planificación Sanitaria, Ministerio de Salud, Chile. 2Departamento Metodologías, División Evaluación Social de Inversiones, Ministerio de Desarrollo Social, Chile. ${ }^{a}$ Economista. Master en Economía (Boston University), Magíster en Gestión y Políticas Públicas (Universidad de Chile). ${ }^{b}$ Economista. MSc. in International Health Policy-Health Economics (London School of Economics and Political Science), Magister en Economía mención Políticas Pública (Universidad Católica de Chile).

Este artículo no contó con ningún tipo de apoyo financiero. Los autores declaran no tener conflictos de intereses. Este artículo fue sometido a revisión por pares.

Correspondencia a: Sergio Poblete Vargas.

Mac Iver 541, Santiago Centro. Eergio.poblete@minsal.c

\section{Determinación de prioridades para la evaluación de tecnologías sanitarias}

\author{
SERGIO POBLETE-VARGAS ${ }^{1, a}$, CARLA CASTILLO-LABORDE $^{2, \mathrm{~b}}$
}

The aim of this article is to analyze the different approaches of priority setting for health technology assessments (HTA). First, the paper identifies the reasons that make necessary to establish priorities and its importance for the success of the HTA models. Second, it studies the main stages that consider the determination of priorities based on the analysis of the models currently used by HTA agencies of developed countries. In the third place, the article describes the different criteria, methods of scoring and deliberation bodies included in the mechanism of priority setting of those agencies. Finally, the paper concludes mentioning lessons from the international experience that potentially can be an input for the design of a model of priority setting for HTA in our country.

(Rev Med Chile 2014; 142 (S1): S 22-26)

Key words: Decision Support Techniques; Health Priorities; Technology Assessment, Biomedical.

4 n todos los sistemas de salud se confronta el desafío de que los recursos no son suficientes para proporcionar todos los servicios que potencialmente podrían beneficiar a la población, por lo que es inevitable decidir qué servicios proveer y quiénes serán los beneficiarios ${ }^{1}$. Este dilema también se presenta en el ámbito de la investigación en salud donde la priorización no resulta ser un tema nuevo. De hecho, existen diversos mecanismos reportados en la literatura para el levantamiento de necesidades de investigación y fijación de prioridades, de manera de asignar los recursos para investigación a los temas que aportarán en la toma de decisiones ${ }^{2}$. Esta preocupación es también extrapolable al caso de la Evaluación de Tecnologías Sanitarias (ETESA), disciplina que ha cobrado una creciente importancia en las últimas décadas, lo que se ha reflejado en la instauración de una serie de programas de ETESA en el ámbito internacional, especialmente en países desarrollados.

Los procesos ETESA, dependiendo del país, se han insertado en una amplia variedad de ins- tituciones como organismos públicos, privados y entes reguladores. Estas organizaciones enfrentan una gran demanda por evaluaciones que exceden con creces la disponibilidad de recursos ${ }^{3}$. Por tanto, las agencias de ETESA también deben determinar prioridades al momento de decidir qué tecnologías serán sometidas a evaluación.

Cabe resaltar que un adecuado mecanismo de priorización es fundamental para que las agencias sean capaces de lograr sus objetivos. Esto porque la utilidad de la información generada por el proceso ETESA para la toma de decisiones depende críticamente de la relevancia de los tópicos seleccionados para ser sometidos a evaluación ${ }^{4}$.

El presente artículo revisa los aspectos centrales de los procesos de priorización analizando las razones que explican la necesidad de determinar prioridades y describiendo los modelos de priorización utilizados por agencias ETESA en el ámbito internacional con el fin de extraer lecciones que potencialmente puedan ser utilizadas en la generación de mecanismos de selección de prioridades en Chile. 


\section{Razones para determinar prioridades para evaluar tecnologías sanitarias}

El tema de la priorización de las tecnologías a evaluar es recogido en el cuarto principio establecido por Drummond (2008) respecto a las agencias o a los procesos ETESA ${ }^{5}$. En este principio se establece que debe existir un sistema explícito para seleccionar prioridades para ETESA, ya que al no ser todas las tecnologías evaluadas, pueden existir distorsiones en la decisión acerca de la inversión y el uso de recursos. $\mathrm{Al}$ respecto, se pueden distinguir tres argumentos que justifican un proceso sistemático de selección de prioridades:

\section{Recursos limitados}

Dada la gran cantidad de tecnologías que potencialmente pueden ser evaluadas, ningún país tiene los recursos suficientes para realizar todas las evaluaciones que idealmente requiere ${ }^{6}$.

\section{Eficiencia (value for money)}

Los promotores de la priorización de las ETESA señalan que ésta puede mejorar la eficiencia del uso de los limitados recursos con que se cuenta al alinear la investigación con los objetivos que persiguen las autoridades de salud ${ }^{4}$. Es decir, la priorización facilita que la información generada por las evaluaciones de tecnologías sea relevante para los tomadores de decisión.

En el mismo sentido, los procesos de selección de tópicos de evaluación bien diseñados permitirían maximizar el retorno (payback) de las investigaciones ETESA no sólo en la toma de decisión, sino que en un sentido más amplio que tiene que ver con el impacto de la información en los resultados de salud de la población ${ }^{6}$. Al respecto, existen estudios que estiman si el retorno de ciertas evaluaciones compensan los costos de llevarlas a cabo ${ }^{7}$.

\section{Transparencia}

Una adecuada priorización permite mejorar la transparencia y rendición de cuentas (accountability) de los sistemas de salud y de las agencias ETESA. La transparencia permite que los productos ETESA sean vistos como útiles y legítimos ${ }^{8}$. En efecto, sistemas de priorización inconsistentes o no transparentes puede generar escepticismo y resistencia en los tomadores de decisión y otras personas que se ven afectadas por las conclusiones de ETESA. En consecuencia, contar con un sistema de priorización claro y explícito es una condición para asegurar la legitimidad de un proceso ETESA. Cierto nivel de transparencia es necesario para que el proceso de priorización sea abierto, sistemático y sin sesgos?.

A pesar de las razones anteriores para contar con sistemas de priorización explícitos y transparentes, tradicionalmente éstos han sido procesos informales y sesgados por grupos de interés ${ }^{4}$. Por otro lado, existe evidencia de que la información generada por las ETESA puede no ser del todo útil para los tomadores de decisión debido a que se evalúan tecnologías no prioritarias ${ }^{10}$. Lo anterior ha llevado a los investigadores a proponer modelos de priorización y a las agencias ETESA a institucionalizar y reformar los procesos de priorización.

\section{Proceso de selección de tecnologías}

$\mathrm{Al}$ revisar los modelos utilizados por diversas agencias ETESA se puede concluir que no existe una fórmula única para seleccionar prioridades. Las agencias ETESA han implementado diversas instancias y mecanismos de priorización dependiendo de los objetivos, institucionalidad y contexto sobre los cuales se realizan las evaluaciones.

Cabe señalar que la diversidad de tópicos a ser evaluados, la urgencia de los tomadores de decisión y otros factores afectan los beneficios de utilizar enfoques sistemáticos y cuantitativos. Sin embargo, existen crecientes esfuerzos por formalizar y hacer más objetivos los mecanismos que utilizan agencias ETESA para seleccionar prioridades.

En términos generales, al establecer un sistema de priorización se deben definir los siguientes aspectos ${ }^{11}$ :

- Cuán sistemático y explícito será el enfoque de determinación de prioridades.

- En qué medida serán aceptados insumos externos e intervenciones de actores no directamente responsables del proceso de priorización (individuos, grupos y organizaciones).

- El peso relativo que se le dará a los puntos de vista de quienes usarán los resultados y quienes desarrollaran las evaluaciones.

- Hasta qué punto el establecimiento de prioridades será transparente y sujeto al escrutinio público.

- El nivel de esfuerzos y recursos que se destinarán al proceso de fijación de prioridades. 
Figura 1. Etapas del Proceso de Selección de Tecnologías. Fuente: Elaborado en base a revisión realizada por Pinson et al., $(2011)^{8}$.

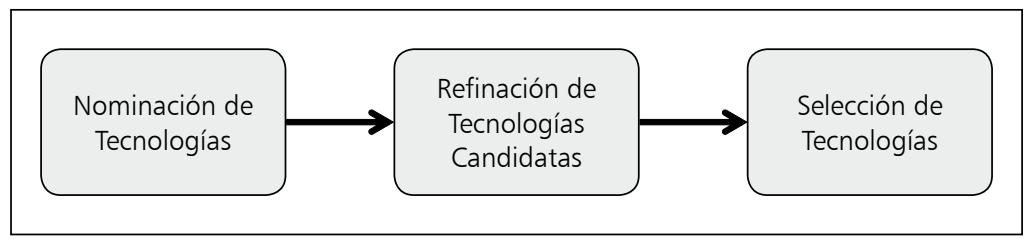

La Figura 1 muestra un esquema estilizado acerca de las distintas etapas que usualmente consideran los procesos de selección de tecnologías a evaluar que usan agencias de países desarrollados.

\section{Nominación de tecnologías}

Corresponde a la etapa de recepción de tópicos y/o tecnologías sobre los cuales se solicita realizar una evaluación. Esta etapa puede ser abierta o cerrada al público. Un ejemplo de un sistema abierto al público es Australia, cuya agencia recibe solicitudes de evaluación de parte de cualquier ciudadano $\mathrm{u}$ organismo privado a través de su página web. En el caso de los sistemas cerrados, la nominación se realiza de forma interna. Esto último se produce en Minnesota, cuya agencia recibe requerimientos de evaluación sólo de parte de las autoridades estatales que administran los servicios de salud ${ }^{8}$.

Cabe mencionar que en los últimos años se han implementado sistemas de monitoreo de nuevas tecnologías que complementan los procesos de nominación a través de la identificación de tecnologías emergentes que potencialmente pueden generar un impacto significativo en los sistemas de salud (en inglés conocidos como Health Care Technology Horizon Scanning). Al respecto, destaca EuroScan que consiste en una iniciativa de colaboración entre las agencias ETESA europeas en que se comparte información sobre nuevas tecnologías de salud ${ }^{12}$.

\section{Refinación de tecnologías candidatas}

Esta etapa es más frecuente cuando se utilizan procesos de nominación abiertos. El objetivo principal es clasificar las tecnologías propuestas en las áreas de trabajo de las distingas agencias. Por ejemplo, en Australia y Canadá se establece una relación con el solicitante para refinar y reorientar la pregunta y el ámbito de la evaluación. Por otro lado, en Inglaterra y Suecia se realiza una búsqueda rápida de literatura para determinar la factibilidad de evaluación ${ }^{8}$.

\section{Selección de tecnologías}

Corresponde a la etapa de priorización propiamente tal. El resultado de esta etapa es un listado de tecnologías y/o áreas prioritarias. De la revisión de experiencias internacionales, se puede concluir que se combinan modelos de carácter cuantitativo e instancias deliberativas.

\section{Modelos de selección de tecnologías}

\section{Criterios involucrados en la priorización}

La Tabla 1 exhibe los criterios identificados en una revisión sistemática de métodos de priorización realizada por Noorani et al. $(2007)^{3}$. Es conveniente aclarar que estos criterios son utilizados como una lista de verificación (check list) y no se debe interpretar que se realizan estudios específicos, lo cual constituiría un estudio ETESA en sí.

Tabla 1. Criterios de priorización prevalentes en agencias ETESA

\begin{tabular}{lcc} 
Criterios & $\begin{array}{c}\text { No de agencias } \\
\text { usando este } \\
\text { criterio } \\
\text { (\% del total) }\end{array}$ \\
Impacto clínico & $11(100)$ \\
Impacto económico & 10 & $(91)$ \\
Carga de enfermedad & 7 & $(64)$ \\
Impacto presupuestario & 6 & $(55)$ \\
Evidencia disponible & 5 & $(45)$ \\
Nivel esperado de interés & 5 & $(45)$ \\
Oportunidad de revisión & 4 & $(36)$ \\
Variación en tasas de uso & 3 & $(27)$ \\
Naturaleza controversial de tecnología & 2 & $(18)$ \\
Implicancia éticas, legales y psicosociales & 2 & $(18)$ \\
Alternativas de tratamiento existentes & 1 & $(9)$ \\
\hline
\end{tabular}

Fuente: Noorani et al. (2007)3. 


\section{Sistemas de puntaje o modelos cuantitativos}

La experiencia pionera en este tipo de modelos fue realizada a principios de los noventa por el Instituto de Medicina de Estados Unidos que propuso considerar ciertos criterios y ponderarlos para obtener un puntaje que dé cuenta de la prioridad para el sistema de salud de determinada tecnología. Este sistema de rankings actualmente es utilizado en España (Agencia Vasca y Catalana) y Canadá considerando criterios y ponderaciones adaptados a su realidad ${ }^{13}$. El puntaje obtenido es utilizado como un insumo para discusión sobre prioridades pues en estas agencias la decisión final acerca qué tecnología evaluar se toma en un proceso deliberativo.

\section{Instancias deliberativas}

La selección final respecto de las tecnologías a evaluar generalmente pasa por instancias deliberativas conformadas por organismos gubernamentales (como los Ministerios de Salud), las juntas directivas de las agencias, y/o sus comités asesores, los cuales dependiendo del país pueden tener representantes de diversos sectores: gobiernos locales, academia, profesionales de la salud, pacientes, etc. Dichas instancias reciben las propuestas de temas identificados y priorizados a partir de los criterios definidos y/o de los métodos cuantitativos, para discutir y finalmente recomendar (e incluso decidir) sobre los tópicos priorizados ${ }^{8}$. Un ejemplo es el caso de Canadá (CADTH), en que se entrega al Comité Asesor información resumida para cada tecnología propuesta para ser evaluada, incluyendo la clasificación de los criterios y su conversión a puntajes y rankings, información de las capacidades instaladas para llevar a cabo la potencial evaluación y otra información adicional relevante. Con esta información se le solicita al Comité deliberar, en una reunión presencial, con el objetivo de asesorar a la agencia respecto de las prioridades de investigación ${ }^{14}$.

\section{Conclusiones}

La institucionalización de las ETESA permite, entre otras cosas, que la toma de decisiones respecto de la asignación de recursos en salud sea más transparente y sistemática. Para que esta premisa se cumpla, un aspecto fundamental está relacionado con la selección de los temas que se abordarán en las evaluaciones, ya que la revisión o no de un tema determinará si este puede acceder a una potencial consideración futura, sea ésta favorable o no.

En este contexto, la forma en que se determinan las prioridades para la evaluación de tecnologías sanitarias juega un rol crucial en el desempeño de la institucionalidad.

El presente artículo revisa algunos modelos de priorización reportados en la literatura y utilizados por agencias de ETESA actualmente existentes. La revisión da cuenta de procesos menos o más estructurados, desde el establecimiento de criterios, hasta la utilización de puntajes para la ponderación de dichos criterios; y el involucramiento de diversos actores en instancias menos o más participativas. Se identifica una tendencia en las agencias revisadas hacia la explicitación de las instancias y actores que tienen un rol en la determinación de prioridades. Adicionalmente, se promueven mecanismos más inclusivos con el objetivo de legitimar el establecimiento de prioridades.

Cualquiera sea el modelo que se implemente, el avanzar hacia procesos menos discrecionales aportará a la validación del proceso de evaluación de tecnologías sanitarias, y finalmente, al proceso de toma de decisiones y formulación de políticas en salud.

\section{Referencias}

1. Peacock S, Richardson J, Carter R. Setting priorities in South Australian Community Health II: Marginal Analysis of Mental Health Services. Research Report. Centre for Health Program Evaluation; 1997.

2. Ranson M, Bennett S. Priority setting and health policy and systems research. Health Res Policy Syst 2009; 7: 27.

3. Noorani HZ, Husereau DR, Boudreau R, Skidmore B. Priority setting for health technology assessments: a systematic review of current practical approaches. Int J Technol Assess Health Care 2007; 23 (3): 310-5.

4. Sassi F. Setting priorities for the evaluation of health interventions: when theory does not meet practice. Health Policy 2003; 63 (2): 141-54.

5. Drummond MF, Schwartz JS, Jönsson B, Luce BR, Neumann PJ, Siebert U, et al. Key principles for the improved conduct of health technology assessments for resource allocation decisions. Int J Technol Assess Health Care 2008; 24 (3): 244-58; discussion 362-8.

6. Davies L, Drummond M, Papanikolaou P, Prioritizing investments in health technology assessment. Can we 
assess potential value for money? Int J Technol Assess Health Care 2000; 16(1): 73-91.

7. Drummond MF, Davies LM, Ferris FL 3rd, Assessing the costs and benefits of medical research: the diabetic retinopathy study. Soc Sci Med 1992; 34 (9): 973-81.

8. Pinson N, Thielke A, King V. Health Tecnology Assessment Rapid Review. Center for Evidence-based Policy: Portland; 2011.

9. Sorenson C, Drummond M, Kanavos P. Ensuring value for money in health care: the role of health technology assessment in the European Union. Observatory Studies Series $N^{\circ} 11$, WHO Regional Office Europe; 2008.

10. Neumann PJ, Rosen AB, Greenberg D, Olchanski NV, Pande R, Chapman RH, et al. Can we better prioritize resources for cost-utility research? Med Decis Making 2005; 25 (4): 429-36.

11. Henshall C, Oortwijn W, Stevens A, Granados A, Banta
D. Priority Setting for Health Technology Assessment: Theoretical Considerations and Practical Approaches: A paper produced by the Priority Setting Subgroup of the EUR-ASSESS Project. Int J Technol Assess Health Care 1997; 13 (02): 144-85.

12. Gutierrez-Ibarluzea I, Simpson S, Benguria-Arrate G; Members of EuroScan International Network. Early awareness and alert systems: an overview of EuroScan methods. Int J Technol Assess Health Care 2012; 28 (3): 301-7.

13. Berra S, Sánchez E, Pons JM, Tebé C, Alonso J, Aymerich $M$. Setting priorities in clinical and health services research: properties of an adapted and updated method. Int J Technol Assess Health Care 2010; 6 (2): 217-24.

14. Husereau D, Boucher M, Noorani H, Priority setting for health technology assessment at CADTH. Int J Technol Assess Health Care 2010; 26 (3): 341-7. 